\title{
Comparison of Complex-Valued Independent Component Analysis Algorithms for EEG Data
}

\author{
Ali Al-Saegh \\ Computer Engineering Department \\ University of Mosul \\ Mosul, Iraq \\ ali.alsaegh@uomosul.edu.iq
}

\begin{abstract}
Independent Component Analysis (ICA) has been successfully applied to a variety of problems, from speaker identification and image processing to functional magnetic resonance imaging (fMRI) of the brain. In particular, it has been applied to analyze EEG data in order to estimate the sources form the measurements.

However, it soon became clear that for EEG signals the solutions found by ICA often depends on the particular ICA algorithm, and that the solutions may not always have a physiologically plausible interpretation. Therefore, nowadays many researchers are using ICA largely for artifact detection and removal from EEG, but not for the actual analysis of signals from cortical sources. However, a recent modification of an ICA algorithm has been applied successfully to EEG signals from the resting state. The key idea was to perform a particular preprocessing and then apply a complexvalued ICA algorithm.

In this paper, we consider multiple complex-valued ICA algorithms and compare their performance on real-world resting state EEG data. Such a comparison is problematic because the way of mixing the original sources (the "ground truth") is not known. We address this by developing proper measures to compare the results from multiple algorithms. The comparisons consider the ability of an algorithm to find interesting independent sources, i.e. those related to brain activity and not to artifact activity. The performance of locating a dipole for each separated independent component is considered in the comparison as well.
\end{abstract}

Our results suggest that when using complex-valued ICA algorithms on preprocessed signals the resting state EEG activity can be analyzed in terms of physiological properties. This reestablishes the suitability of ICA for EEG analysis beyond the detection and removal of artifacts with real-valued ICA applied to the signals in the time-domain.

Index Terms-Blind source separation, cocktail party problem, complex, dipole localization, EEG, electroencephalography, ICA, independent component analysis, inverse problem, real, signal processing.

\section{INTRODUCTION}

Independent component analysis (ICA) is a mathematical model for estimating underlying components in a high-density mixed data with the aim of estimating components as independent as possible [1]. The blind source separation problem (BSS), sometimes called the blind signal separation problem, is a general form of ICA. BSS is about estimating the original signals (components) from their observed mixture data depending on several assumptions about the mixing process, the word "blind" denotes that it is known very little if nothing about the original components. BSS has been used in a vast number of situations in which observed mixed data is originating such as convoluted mixtures of images [2], psychometric measurements, stock market indicators [1], and artifacts removal from EEG recordings [3], etc.

The cocktail party problem is one of the most used examples for demonstrating the problem of finding hidden variables in the mixed data. In this case, we can imagine that we have three persons sitting in a room and talking to each other simultaneously, also there are three microphones fixed at different positions in the room recording mixed voices from the three persons. The recorded voice signals are of course not clear to a listener. 
In this paper considers multiple complex-valued ICA algorithms and compare their performance on real-world resting state EEG data. Such a comparison is problematic because the way of mixing the original sources (the "ground truth") is not known. We address this by developing proper measures to compare the results from multiple algorithms.

The mathematical ICA model is given in (1) where $x_{1}, \cdots, x_{n}$ are the observed mixed signals, $s_{1}, \cdots, s_{n}$ are the unrelated original signals, and $a_{i j}$ are some real coefficients that represent the weights of mixing the original signals [4]. This equation can be written in a vector-matrix representation as in (2). There are several approaches that are used for estimating the unmixing matrix $\boldsymbol{W}$ (an estimation for the inverse of the mixing matrix $\boldsymbol{A}^{-1}$ ) such that $\boldsymbol{y}=\boldsymbol{W} \boldsymbol{x}$ where $y_{i}$ are the estimated variables of the original variables $s_{i}$ up to scaling and permutation of the rows of $\boldsymbol{W}$. All of the ICA algorithms that have been used in this study are presented in section III with their principles of estimating the Independent Components (ICs).

$$
\begin{gathered}
x_{i}(t)=a_{i 1} s_{1}(t)+a_{i 2} s_{2}(t)+\cdots+a_{i n} s_{n}(t) ; i=1, \ldots, n \\
\boldsymbol{x}=\boldsymbol{A} \boldsymbol{s}
\end{gathered}
$$

The German psychiatrist Hans Berger was the pioneer of the human electroencephalogram (EEG) when first discovered it in 1929 [5]. This technique records the electrical activities generated by the brain via a number of electrodes (channels) fixed on the scalp. The EEG recording technique is most suitable in cases where high temporal resolution is required. The main contributing brain sources to the EEG recorded data are emitted from different small places within the brain called patches. Those patches are normally modeled as electrical dipoles. Modeling the dipoles requires a solution for both volume conductor model of the brain and the behavior of current sources in the brain. This can be achieved by solving the Poisson's equation as it relates the current sources and potentials formed in the volume conductor [6]. Ideally, the process of locating sources within the brain (dipoles) using EEG signals involves the inverse solution of
Poisson's equation for parceling the brain, then followed by an iterative procedure of locating the sources.

\section{Complex-VAlued ICA}

Complex-valued signals often occur in applications such as communications, radar, images, and biomedicine. Complex-valued ICA has been widely dealt with in research and practical work. In this study, we were interested in analyzing rest-state EEG data using complex-valued ICA and then comparing the performance of different algorithms based on some criteria.

Analyzing complex-valued EEG signals through the frequency-domain representation of the signals have advantages over time-domain as several scientists have shown that EEG activities have specific features in different frequency bands which might be related to different physiological activities [7]. Moreover, real-valued ICA does not consider the propagation delay to the recording electrodes and that each oscillatory signal is recorded at the same phase. This leads to assigning a single current dipole for only one source, whereas there might exist sources that are spatially more distributed which are recorded with small a time difference and, therefore, assigning several dipoles for each of them is more reasonable. Those spatially extended sources can also be gathered in a single component rather than having them separated into different components [8]. This requires modeling the mixing matrix as complex-valued rather than real-valued mixing matrix as in the case of real-valued ICA. We use the short-time Fourier transform (STFT) in order to decompose the EEG signals into different spectral bands then investigate the oscillatory activity. Based on the common property of the linear time-filtering, such decomposition does not in any way alter the linear mixing model and its specifications as well [8]. Hence, further analysis and results are considered trustworthy.

The classical linear mixing model for EEG data is given in (3), indicating by $x_{c, \tau}$ the EEG data matrix where $c$ is the channel index and $\tau$ is the time index, $s_{p}$ are the source signals (or the ICs) where $p$ is an index for the IC. 


$$
x_{c, \tau}=\sum_{p=1}^{P} a_{c, p} s_{p, \tau}
$$

STFT is applied to the raw EEG data with fixed spaced temporal indices $t_{\tau}$ and then the new resulting matrix $x_{c, t f}$ is adopted for further analysis. This step transforms the two-dimensional data matrix into another two-dimensional matrix but with the three indices: $c$ is the channel index, $t$ is the STFT's window index with a lower resolution than $\tau$, and $f$ is the Fourier coefficient index within the Fourier transformed window. The short-time Fourier transformed windows taken from each row in the raw data matrix are arranged one-by-one in the corresponding row of the new matrix, therefore, the transformed matrix is still with two-dimensions. Thus, the linear mixing model after the STFT of the data becomes as stated in (4). The data matrix $\boldsymbol{X}$ in the new model is complex-valued by the definition of the Fourier transform, whereas the matrix of mixing coefficients $A$ is still real-valued as in (3). However, the process of modeling the very small delays $\delta(c, p)$ of recording brain signals at different electrodes represents a transformation of the realvalued mixing matrix to a complex-valued one. Modeling these delays means that a source signal is observed in different electrodes with different phases. The derivation of the complete complexvalued mixing model (5) is given in [8]. Each of the derived mixing parameters in this equation is complex-valued, its real part is the old real-valued mixing coefficients $a_{c, p}$ and its imaginary part is the new term $e^{\left(2 \pi i f_{0}(p) \delta(c, p)\right)}$.

$$
\begin{gathered}
x_{c, t f}=\sum_{p=1}^{P} a_{c, p} s_{p, t f} \\
x_{c, t f}=\sum_{p=1}^{P} a_{c, p} e^{\left(2 \pi i f_{0}(p) \delta(c, p)\right)} s_{p, t f}
\end{gathered}
$$

The Fourier coefficients retrieved after applying the STFT to the raw EEG data may be sparsely distributed when most of the coefficients corresponding to some frequency bands are with zero energy and only a few of them are with nonzero. This gives the analysis of complex-valued EEG signal more advantages in finding sources of oscillatory activity that belong to the brain but not to the artifacts [7], [8].

\section{Materials ANd Methods}

\section{A. The Complex-Valued ICA Algorithms}

A large number of ICA algorithms have been developed for analyzing data in different fields. The ICA algorithms can be generally classified according to the principal they are based on such as negentropy or nongaussianity maximization, maximum-likelihood estimation, or mutual information minimization. In this study, we selected five of the recent algorithms based on different estimating approaches. Here, we present the algorithms in a systematic manner by describing the contrast function of the algorithm and the estimation procedure and its optimization condition. The algorithms with their references are: Adaptive Complex Maximization of Nongaussianity (ACMN) [9], Complex ICA by Entropy Bound Minimization (cICA-EBM) [10], Complex FastICA (c-FastICA) [8], Kurtosis Maximization using Fixed-point update (KM-F) [11], and Generalized Uncorrelating Transformation (GUT) [12]. Please note that all given mathematical equations that are related to the algorithms can be found in those references.

\section{1) Adaptive Complex Maximization of} Nongaussianity $(A-C M N)$

Complex Maximization of Nongaussianity $(\mathrm{CMN})$ algorithm uses the contrast function given in (6), where $\boldsymbol{x}$ is the vector of observed data, $\boldsymbol{w}$ is an unmixing vector, and $H$ is the Hermitian transpose of $\boldsymbol{w}$. The function $G(z)=z^{K} / \sqrt{2 K}$ is used in order to derive the adaptive $\mathrm{CMN}(\mathrm{A}-\mathrm{CMN})$ algorithm by submitting it into (6) and get the nonlinearity given in (8), $z$ is a complex random variable and $K$ is the number of source variables.

$$
J(\boldsymbol{w})=E\left\{\left|G\left(\boldsymbol{w}^{H} \boldsymbol{x}\right)\right|^{2}\right\}
$$




$$
\begin{gathered}
\quad J(z)=\frac{z^{K}}{\sqrt{2 K}}\left(\frac{z^{K}}{\sqrt{2 K}}\right)^{*} \\
=e^{K \log (z)} e^{K \log \left(z^{*}\right)} / 2 K \\
=e^{K \log |z|+j \arg (z)} e^{K \log |z|-j \arg (z)} / 2 K \\
=|z|^{2 K} / 2 K
\end{gathered}
$$

The ideal weight for the CMN that maximizes the contrast function under the unit norm restriction is given as:

$$
\boldsymbol{w}_{\text {opt }}=\arg \max _{\|\boldsymbol{w}\|^{2}=1} E\left\{\left|G\left(\boldsymbol{w}^{H} \boldsymbol{x}\right)\right|^{2}\right\}
$$

In CMN algorithm, every source $\hat{s}_{k}$ is estimated individually by finding a vector $\boldsymbol{w}$ such that

$$
\hat{s}_{k}=\boldsymbol{w}_{k}^{H} \boldsymbol{x}=\boldsymbol{w}_{k}^{H} \boldsymbol{A} s=\boldsymbol{q}_{k}^{H} s
$$

where $\boldsymbol{A}$ is the mixing matrix, and $\boldsymbol{q}_{k}=$ $\left[0, \ldots, q_{k}, 0, \ldots\right]^{T}$.

The fixed-point update for the optimization problem which is defined in (9) is given in (11), where $G$ is defined in (6), $g$ is the derivative of $G$, and $y=\boldsymbol{w}^{H} \boldsymbol{x}$.

$$
\begin{aligned}
\boldsymbol{w}^{n+1}=-E\left\{G^{*}\right. & (y) g(y) \boldsymbol{x}\} \\
& +E\left\{g(y) g^{*}(y)\right\} \boldsymbol{w}^{n}+E\left\{\boldsymbol{x} \boldsymbol{x}^{\boldsymbol{T}}\right\} \\
& +E\left\{G^{*}(y) g^{\prime}(y)\right\}\left(\boldsymbol{w}^{n}\right)^{*}
\end{aligned}
$$

We do not move into all the details of deriving the whole algorithm which can be found in the stated reference. The A-CMN algorithm can be summarized in two steps: the first one is the estimation of $K$ using (13), and the second one is to use the nonlinearity in the fixed-point algorithm (11); where $p$ is a shape function for determining the distribution type as supergaussian, subgaussian, or Gaussian.

$$
\begin{gathered}
p^{n+1}=p^{n}-\left(\frac{\partial \log E\left\{y^{2}\right\}}{\partial p}\right)^{-1} \log E\left\{y^{2}\right\} \\
K^{n+1}=\frac{p^{n+1}}{2}
\end{gathered}
$$

\section{2) Complex ICA by Entropy Bound Minimization (cICA-EBM)}

The cICA-EBM algorithm uses the contrast function given in (14), where $N$ is the number of complex sources $s(t)=$ $\left[s_{1}(t), \ldots, s_{N}(t)\right], I\left(z_{1} ; \ldots ; z_{N}\right)$ represents the mutual information among $N$ random variables $z_{N}$ to be estimated where $n=1, \ldots, N, \boldsymbol{W}$ is the unmixing matrix, $H\left(z_{N}\right)$ is the entropy of the $n$th source, and $H(x)$ is the entropy of observations.

$I\left(z_{1} ; \ldots ; z_{N}\right)=\sum_{n=1}^{N} H\left(z_{N}\right)-2 \log |\operatorname{det}(\boldsymbol{W})|-H(x)$

The optimization problem given in (15) is a function of $\boldsymbol{w}_{n}$. Where $\widehat{H}\left(z_{n}\right)$ is the entropy estimator [10], $z_{n}=\boldsymbol{w}_{n}^{H} \boldsymbol{x}, \quad C$ is a quantity independent of $\boldsymbol{w}_{n}$, and $-2 \log \left|h_{n}^{H} \boldsymbol{w}_{\boldsymbol{n}}\right|$ is a function that attempts to constrain $\boldsymbol{w}_{n}$ to be orthogonal to all the other row vectors of the unmixing matrix $\boldsymbol{W}$.

$$
\min _{\left\|\boldsymbol{w}_{n}\right\|=1} J_{n}\left(\boldsymbol{w}_{n}\right)=\widehat{H}\left(z_{n}\right)-2 \log \left|h_{n}^{H} \boldsymbol{w}_{\boldsymbol{n}}\right|+C
$$

The line search algorithm is given as:

$$
\begin{gathered}
\boldsymbol{w}_{n}^{+}=\boldsymbol{w}_{n}-\mu \boldsymbol{u}_{n} \\
\boldsymbol{w}_{n}^{[n e w]}=\frac{\boldsymbol{w}_{n}^{+}}{\left\|\boldsymbol{w}_{n}^{+}\right\|}
\end{gathered}
$$

where $\mu>0$ is the step size, and $\boldsymbol{u}_{n}$ is computed using the normalized projected conjugate gradient in (19).

$$
\begin{gathered}
\boldsymbol{u}_{n}^{+}=\frac{\partial J_{n}\left(\boldsymbol{w}_{n}\right)}{\partial \boldsymbol{w}_{n}^{*}}-\mathfrak{R}\left\{\boldsymbol{w}_{n}^{H} \frac{\partial J_{n}\left(\boldsymbol{w}_{n}\right)}{\partial \boldsymbol{w}_{n}^{*}}\right\} \boldsymbol{w}_{n} \\
\boldsymbol{u}_{n}=\frac{\boldsymbol{u}_{n}^{+}}{\left\|\boldsymbol{u}_{n}^{+}\right\|}
\end{gathered}
$$

The line search procedure outlined in (17) is repeated over different row vectors of $\boldsymbol{W}$ until convergence. 


\section{3) Complex FastICA (c-FastICA)}

The c-FastICA algorithm uses the contrast function given in (20). The function $G=(1 / 2) y^{3}$ that is motivated by kurtosis is used as a nonlinear objective function.

$$
J(\boldsymbol{w})=E\left\{G\left(\left|\boldsymbol{w}^{H} \boldsymbol{x}\right|^{2}\right)\right\}
$$

The optimization problem is formulated as:

$$
\boldsymbol{w}_{\text {opt }}=\arg \max _{\|\boldsymbol{w}\|^{2}=1} E\left\{\left|G\left(\boldsymbol{w}^{H} \boldsymbol{x}\right)\right|^{2}\right\}
$$

The derived fixed-point update for the constrained optimization problem could be written as:

$$
\begin{aligned}
\boldsymbol{w}_{n+1}=-E\{ & \left.\left(|y|^{2}\right) y^{*} \boldsymbol{x}\right\} \\
& +E\left\{g^{\prime}\left(|y|^{2}\right)|y|^{2}+g\left(|y|^{2}\right)\right\} \boldsymbol{w}_{n} \\
& +E\left\{\boldsymbol{x} \boldsymbol{x}^{T}\right\} E\left\{g^{\prime}\left(|y|^{2} y^{*^{2}}\right)\right\} \boldsymbol{w}_{n}^{*}
\end{aligned}
$$

where $y=\boldsymbol{w}^{H} \boldsymbol{x}$. Symmetric orthogonalization is implemented in this algorithm, such that all sources are estimated in parallel.

\section{4) Kurtosis Maximization using Fixed-point update $(K M-F)$}

The contrast function for this algorithm tries to maximize the complex kurtosis or the fourth-order cumulant of a zero-mean random variable which is defined for random variable $y$ as the real number:

$$
\kappa(y)=E\left\{|y|^{2}\right\}-2\left(E\left\{|y|^{2}\right\}\right)^{2}-\left|E\left\{y^{2}\right\}\right|^{2}
$$

The kurtosis for the unit-norm vectors $\boldsymbol{W}_{t}$ and $\boldsymbol{W}_{t+1}$ is

$$
\begin{aligned}
\Delta \kappa & =\kappa\left(\boldsymbol{W}_{t+1}\right)-\kappa\left(\boldsymbol{W}_{t}\right) \\
& =2 \operatorname{Re}\left\{\left\langle\nabla \mathbf{w} * \kappa \mid \mathbf{w}_{t},\left(\boldsymbol{W}_{t+1}-\boldsymbol{W}_{t}\right)\right\rangle\right\}
\end{aligned}
$$

Given $\boldsymbol{W}_{t}$, we use the Cauchy-Schwarz inequality in order to find $\boldsymbol{W}_{t+1}$ such that $\Delta \kappa$ is maximized:

$$
\left|\left\langle\nabla \mathbf{w} * \kappa \mid \mathbf{w}_{t}, \mathbf{W}\right\rangle\right| \leq\left\|\nabla \mathbf{w} * \kappa \mid \mathbf{w}_{t}\right\|
$$

where the maximum value is achieved if and only if $\boldsymbol{W}_{t+1}=\nabla \mathbf{w} * \kappa\left|\mathbf{w}_{t} /\left\|\nabla \mathbf{w} * \kappa \mid \mathbf{w}_{t}\right\|\right.$, so that the $\mathrm{KM}$ update is given as $\boldsymbol{W}_{t+1}=$ $\nabla \mathbf{w} * \kappa\left|\mathbf{w}_{t} /\left\|\nabla \mathbf{w} * \kappa \mid \mathbf{w}_{t}\right\|\right.$ to maximize $\Delta \kappa$. The complex fixed-point $\mathrm{KM}$ update is formulated as:

$$
\boldsymbol{W} \leftarrow \nabla \mathbf{w} * \kappa\left(\boldsymbol{w}^{H} \boldsymbol{x}\right) \quad \boldsymbol{W} \leftarrow \frac{\boldsymbol{w}}{\|\boldsymbol{W}\|}
$$

\section{5) Generalized Uncorrelating Transformation (GUT)}

The GUT algorithm is considered as an expansion of the whitening conversion process for complexvalued random vectors. It is a second-order ICA model and it is a generalized form of the stronguncorrelating transform (SUT) algorithm that is proposed by Eriksson and Koivunen [13]. GUT aims to find uncorrelated components based upon the estimation of the covariance and pseudo-covariance matrices. The GUT matrix can be calculated by first calculating the square-root matrix $\boldsymbol{B}(\mathbf{z})$ of $\boldsymbol{C}(\mathbf{z})^{\mathbf{- 1}}$, so $\boldsymbol{B}(\boldsymbol{z})^{\boldsymbol{H}} \boldsymbol{B}(\boldsymbol{z})=\boldsymbol{C}(\boldsymbol{z})^{-\mathbf{1}}$ and the whitened data $\boldsymbol{v}=\boldsymbol{B}(\boldsymbol{z}) \boldsymbol{z}$; where $\boldsymbol{C}(\boldsymbol{z})$ is the scatter matrix of the complex random vector $\boldsymbol{z}$. This step represents a whitening for the data such that $\boldsymbol{C}(\boldsymbol{v})=\boldsymbol{I}$.

Then we use a distinct form of the singular valued decomposition namely the Takagi's factorization for the whitened data $\boldsymbol{v}$ such that $\boldsymbol{P}(\boldsymbol{v})=\boldsymbol{U} \boldsymbol{\Lambda} \boldsymbol{U}^{T}$ where $\boldsymbol{P}$ is a spatial pseudo-scatter matrix, $\boldsymbol{U}=\boldsymbol{U}(\boldsymbol{v})$ is the Takagi factor of $\boldsymbol{P}(\boldsymbol{v})$, and $\boldsymbol{\Lambda}$ is the singular values $\lambda_{i}=[\boldsymbol{P}(\boldsymbol{s})]_{i i}$. The whitening transformation GUT matrix is found as $\boldsymbol{W}(\boldsymbol{z})=\boldsymbol{U}(\boldsymbol{v})^{H} \boldsymbol{B}(\mathbf{z})$.

\section{B. Comparison Criteria}

The decision factors within this study are quantification for the separation quality and quantification for the solution of the inverse problem. The comparison criteria we have used do not depend on the ground truth of the ICA model because the concrete structure of the interacting sources in the real EEG data and the way they have been mixed are unknown for us. We emphasize that the assumptions related to the ICA model may not exactly hold because we are trying to estimate the original sources from the real-world and not synthesized data. Following is a brief description of each of the used criteria. 


\section{1) Degree of Separation}

The measure of statistical independence is computed in order to quantify the separation goodness that is achieved by each of the used algorithms. The degree of dependency between each pair of the estimated sources was considered at each frequency $f$ within the adopted frequency band $(0-$ $50 \mathrm{~Hz}$ ) by computing the mean $\rho(f)$ of the absolute values of correlation coefficients $\rho_{i j}(f)$ over all different estimated source pairs $i \neq j$ as shown below:

$$
\begin{gathered}
\rho(f)=\frac{1}{N(N-1)} \sum_{i \neq j} \rho_{i j}(f) \\
\rho_{i j}(f)=\left|\frac{\left\langle\left|s_{i}(T, f)\right|^{2}\left|s_{j}(T, f)\right|^{2}\right\rangle_{T}-\mu_{i}(f) \mu_{j}(f) \mid}{\sigma_{i}(f) \sigma_{j}(f)}\right| \\
\mu_{i}(f)=\left\langle\left|s_{i}(T, f)\right|^{2}\right\rangle_{T} \\
\sigma_{i}(f)=\sqrt{\left\langle\left(\left|s_{i}(T, f)\right|^{2}-\mu_{i}(f)\right)^{2}\right\rangle_{T}}
\end{gathered}
$$

where $\langle.\rangle_{T}$ indicates the expectation which is calculated as the sample average over the number of time points $T$ of the estimated source signal, $\mu_{i}(f)$ and $\sigma_{i}(f)$ are respectively the mean and the standard deviation of the $i$ th component. Equation (28) [1] measures the degree of dependency between each pair of ICs [1]. For independent signals, the index $\rho_{i j}(f)$ reaches its minimum (zero) whereas it reaches its maximum (one) only when the signals are proportional.

\section{2) Measure of Sparseness}

The procedure of ICA estimation always looks for nongaussian sources. In practice, most of the sources are supergaussian or sparse [8]. A random variable is said to be sparse when it is occurring at widely spaced intervals and it is most of the time close to zero. The Fourier coefficients returned after applying the STFT on the raw EEG data can also be sparsely distributed when most of the coefficients corresponding to some frequency bands are with zero energy and only a few of them are with nonzero. Therefore, this measure attains its maximum (one) for those sources with the highest supergaussianity or sparseness. We use the objective function in (31) to measure how much an estimated source $\left(s_{i, t f}\right)$ sparse is [14], where $F$ is the number of STFT's coefficients.

$$
I\left(s_{i}\right)=\frac{1}{T F} \sum_{t, f}-\log \left(1+\left|s_{i, t f}\right|^{2}\right)
$$

\section{3) Residual Variance of Dipole Localization}

The process of decomposing multichannel EEG signals using ICA determines temporally independent components and their projection form on the scalp surface. Whereas each column in the resulting mixing matrix $\left(W^{-1}\right)$ denotes, for an IC, the proportional projection weight at each of the recording electrodes. Charting the elements of these columns to the related electrodes on a head model gives an imaging of the scalp projection for each of the estimated sources. This proposes a favorable approach for solving the inverse problem of EEG, specifically by achieving inverse modeling of the separated IC scalp projection. The most interesting thing is that those IC scalp projections or scalp maps have been found to be more localized or dipole-like on the head model than the raw EEG [3], [15]; which could make it easier to model the corresponding dipoles. Detailed information concerning the inverse problem and modeling of ICs can be found in [15].

During the dipole localization procedure, each of the observed scalp maps is assigned the bestrepresenting dipole(s) located in a 3D head model at some location(s) and orientations. This matching process is solved up to some error or residual variance between the observed scalp map and the scalp projection of the assigned dipole. That error $(E)$ for each IC can be calculated using (32), where each column of $F$ represents the best-fitting dipole for an IC in a 3D head model, and $N$ is the number of estimated sources. The less the value of the residual variance is, the better is the fitted dipole(s) for an estimated component. 


$$
E(j)=\frac{\sum_{i=1}^{N}\left(W^{-1}(i, j)-F(i, j)\right)^{2}}{\sum_{i=1}^{N}\left(W^{-1}(i, j)\right)^{2}}, j=1, \ldots, N
$$

\section{The EEG Datasets}

The used EEG datasets are taken from 105 elderly subjects. The subjects were in resting state, where the eyes were closed and nearly without any movements at the body parts. The length of the signals is around 10 minutes. Some of the datasets were recorded using a head cap equipped with 22 electrodes and the others with 32 electrodes. The amplified EEG signals were sampled and stored at either 250 or 1000 samples per second. We processed all EEG datasets as stated in the following steps:

1) The raw EEG datasets contain channel labels only, we added the international 10-20 standard channel location information [16] to the data as it is necessary for plotting EEG scalp maps in either $2 \mathrm{D}$ or $3 \mathrm{D}$ format or to estimate source locations for data components.

2) Common average reference (CAR) is used as a re-reference for the recorded data in order to obtain plain EEG.

3) STFT is applied with windows of $1 \mathrm{sec}$ duration with half overlapping. Then only coefficients in the range of $(0-50 \mathrm{~Hz})$ were adopted.

4) All the Fourier transformed signals were forced to zero-mean.

5) Then we applied the ICA algorithms.

6) Dipole localization procedure is applied in order to assign one or two dipoles for each of the estimated sources.

7) We used the EEGLAB toolbox as an assistant tool. It is available on (https://sccn.ucsd.edu/eeglab/index.php) for free.

\section{Results}

Following is a presentation of the experimental comparison results, we grouped the results according to the important criteria.

\section{1) Degree of Separation}

One of the important criteria for quantifying the performance of an ICA algorithm is the measure of statistical independence between the estimated sources.

Fig. 1 shows the degrees of separation between the estimated sources (ICs) which are obtained from the five ICA algorithms. Each of the drawn traces represents the dependencies obtained from one algorithm over the frequency band $(0-50 \mathrm{~Hz})$ that is adopted from the Fourier transformed original data prior to the ICA. So that, the separation index at each frequency is computed by averaging the correlations for all different pairs of the estimated sources. Then, the obtained correlation index at each frequency component is averaged over all analyzed EEG signals which are recorded from the different subjects. The black trace represents the amount of dependency between the Fourier transformed data channels, i.e. before applying any of the ICA algorithms.

As mentioned before, the smaller value of the separation index is, the better is the performance of the algorithm. Hence, these results show that all the algorithms, except GUT, were able to separate the observed mixed EEG data into their independent sources with a lower degree of dependency than the exists within the original EEG data channels. It is clear that the performance of GUT was not satisfied as other algorithms. Although the performance of the other algorithms is almost the same however, at some frequency bands both cICA-EBM and A-CMN did slightly better separation than both c-FastICA and KM-F.

\section{2) Component Source Modeling}

The EEG channel locations are considered as useful markers when giving the locations and strengths of emitted signals by the brain patches. However, EEG recordings do not contain any information about the physical locations of those EEG recording channels. The solution to this problem is to inversely model each of the estimated scalp maps as one or more dipoles located in a 3D head model. The solution for the inverse problem has been discussed earlier in this paper, we mentioned that each column in the estimated mixing matrix represents a scalp map.

After analyzing the EEG data with the ICA 


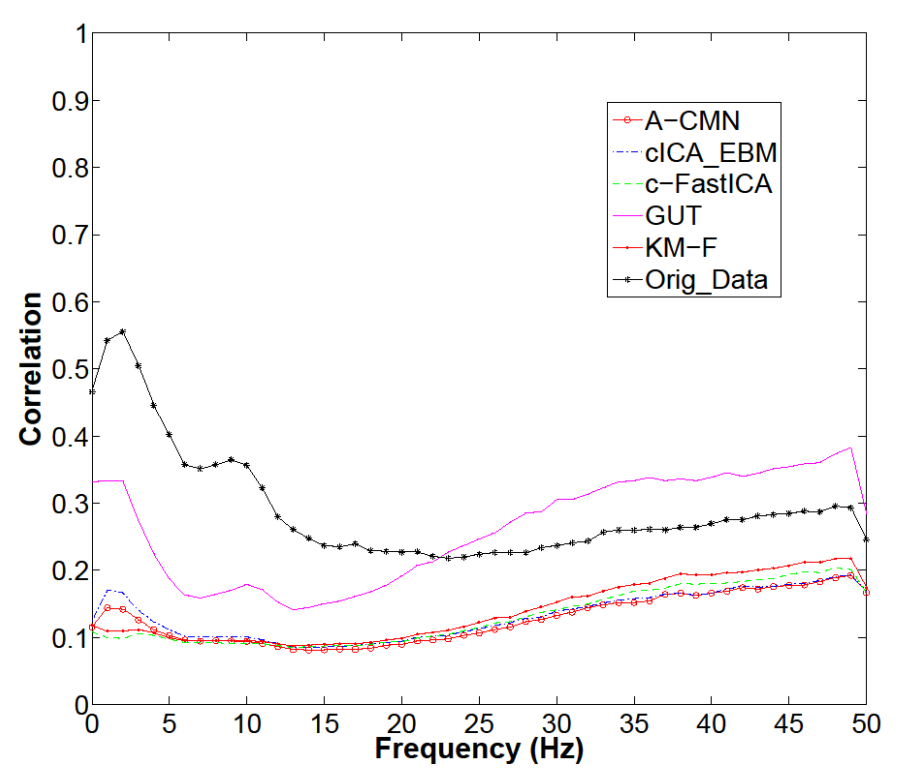

Fig. 1. Statistical dependencies at the frequency band $(0-50 \mathrm{~Hz})$. The degrees of dependency between the estimated sources which are achieved by cICA-EBM, A-CMN, c-FastICA, and KM-F are lower than those exist in EEG data channels; this means that these algorithms were able to separate the mixed EEG data into their original sources. While GUT was able to estimate the original sources at low-frequency bands.

algorithms, we used the EEGLAB Matlab toolbox with the DIPFIT (an EEGLAB plugin) in order to model dipoles for the estimated sources. The available EEG inverse model with the EEGLAB toolbox tries to assign one or two best fitting dipoles for each estimated source.

Fig. 2 shows example results for some of the fitted dipoles with their scalp maps. A three-shell boundary element model for the 3D head model is used for modeling and visualizing the locations of dipoles in the head. The numbers nearby the dipoles represent the index of the estimated IC. The locations and directions of the dipoles indicate the amount of planned electric field on the scalp. Such that, those scalp maps that demonstrate more localized brain activities are assigned single-dipole as it is shown for ICs: 2, 13, 19, 22, 25, and 26. Whereas those scalp maps which somehow demonstrate left-right symmetric activity cannot be accurately modeled with a single-dipole, therefore, they are assigned a dual-dipole as it is the case with ICs: 17 and 27.

Also, the residual variance between the IC scalp map and the dipole scalp projection is shown nearby each scalp map. The values of residual variance for most of the fitted dipoles were close to the minimum predictable level of error, the statistics for that is

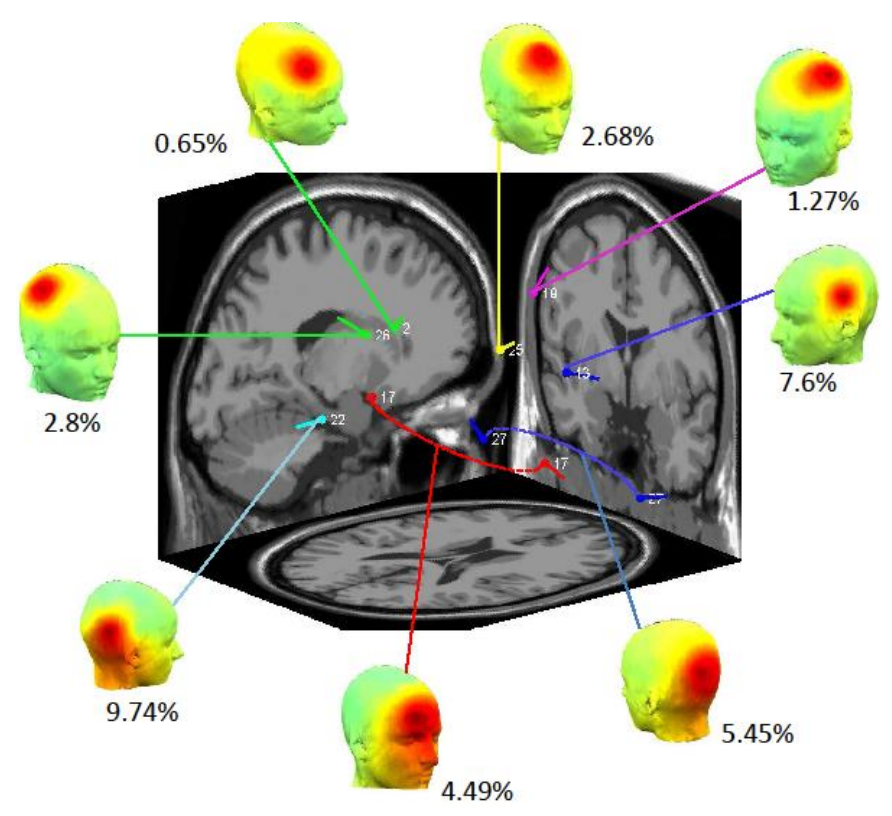

Fig. 2. The 3D scalp maps for the estimated ICs are linked by lines to their respective modeled dipole(s). The dipoles within the 3D head model were found by inversely modeling of those scalp maps. The more localized brain activities are assigned a single-dipole as it is the case with the ICs: 2, 13, 19, 22,25 , and 26; whereas the more distributed brain activities are assigned a dual-dipole as it is the case with the ICs: 17 and 27.

shown in Fig. 3 and will be discussed next. We think that this variance occurs due to the probable drawbacks in the 3D head modeling as a set of conductive spheres (brain, skull, and scalp), also the possible inadequate setting up of the EEG recording cap on the subject's head has a big effect on the dipoles modeling.

Fig. 3 shows statistics concerning the residual variance of the fitted dipoles for all the estimated sources in all analyzed EEG datasets (105 subjects). We notice that around $75 \%$ of the estimated sources were assigned dipole(s) with residual variance of less than $20 \%$, around $20 \%$ of the estimated sources were assigned dipole(s) with residual variance in the range of $20 \%-50 \%$, around $5 \%$ of the estimated sources were assigned dipole(s) with residual variance of greater than $50 \%$, and around $3 \%$ of the estimated sources were failed to converge.

This index shows no big difference in the performance of the different algorithms, and the fitting process was able to assign dipoles with low residual variances to a large number of the estimated sources. We think this is not unexpected results because in the case of resting-state EEG analysis it is more likely to estimate an IC that is representing a brain activity but not an artifact activity. Normally, 


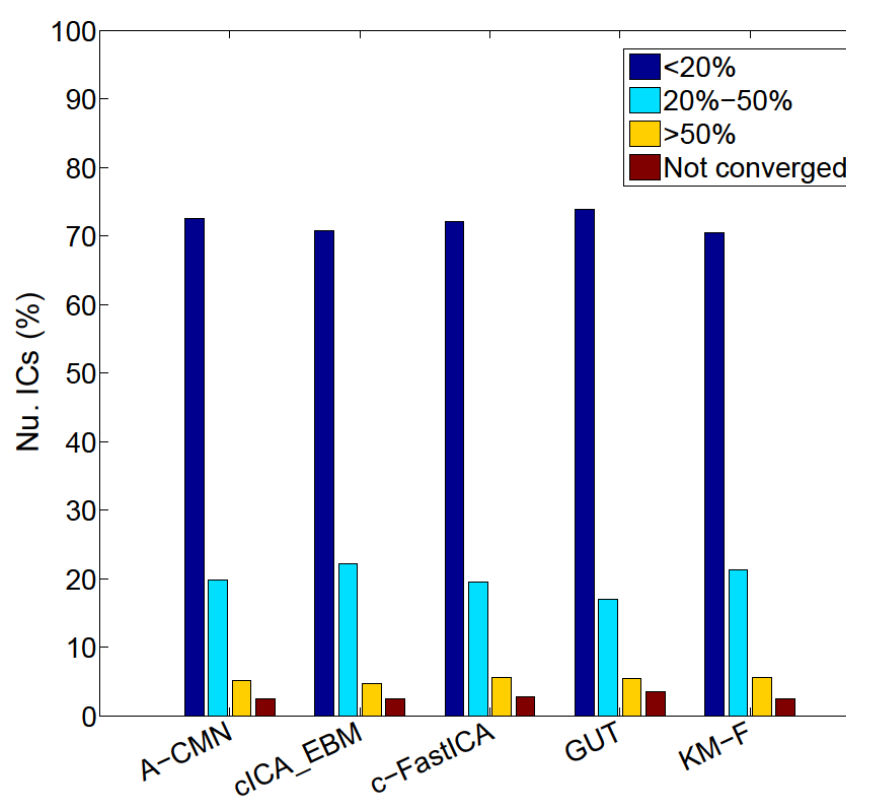

Fig. 3. Residual variance statistics of the fitted dipoles for the estimated ICs. It explains that around 75\% of the estimated ICs were assigned dipoles with residual variance less than $20 \%$, around $20 \%$ of the ICs were assigned dipoles with residual variance $20-50 \%$, around $5 \%$ of the ICs were assigned dipoles with residual variance greater than $50 \%$, and around $3 \%$ of the ICs had failed to converge.

the scalp map for the brain activity is more localized than the one for an artifact activity, and it seems that most of the estimated scalp maps had localized activities which can be easily modeled by the dipole fitting algorithm with even low residual variances.

\section{3) Estimation of Sources}

This section shows the performance of different complex-valued ICA algorithms for estimating hidden sources from high density mixed EEG signals. Based on the sparseness index which was discussed earlier, we present averaged results in order to compare the performance of the algorithms. The aim behind using this measure (sparseness) is to find the most interesting sources, namely those related to brain activity.

Fig. 4 shows the performance of the algorithms in estimating cerebral ICs. The sparseness index was computed for all the estimated sources in all the analyzed EEG datasets and then averaged and normalized overall 22-channel or 32-channel EEG data as shown in the two sub-figures. It is clear from the two plots that there is no big difference in the performance that is achieved by cICA_EBM, ACMN, KM-F, and c-FastICA. However, the cICA_EBM algorithm slightly outperformed the
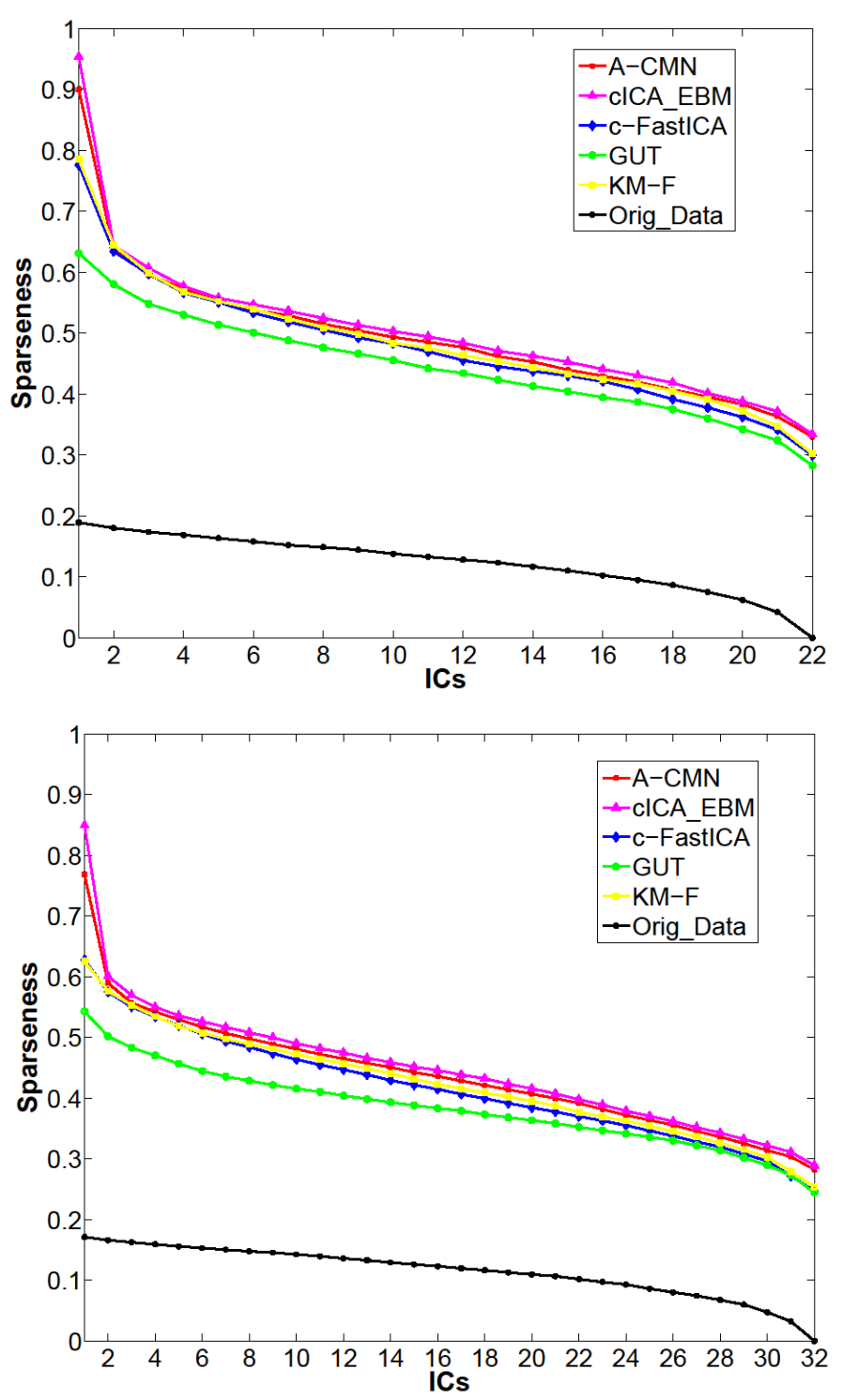

Fig. 4. Sparseness index that is averaged over all analyzed EEG datasets. These are the normalized values returned by the index. The higher the value means the sparser the IC is, which in turn means that the IC is more likely to represent a cerebral activity. The cICA_EBM outperformed other algorithms, and GUT had low performance. The two plots show the consistency of ICA algorithms with the different number of EEG recording channels.

others; and GUT showed the worst performance among the algorithms.

The numeral results which are returned from analyzing all EEG datasets showed no big difference from one dataset to another and since it is impossible to present details of all those results, therefore, in Fig. 5 we show results that are returned from only one of the datasets that is recorded using 32-channel (i.e. 32 sources were estimated). The figure visualizes the performance of the algorithms for estimating all the sources. The upper row in the image (Orig_Data) represents the sparseness of the Fourier transformed original data signals (channels), 


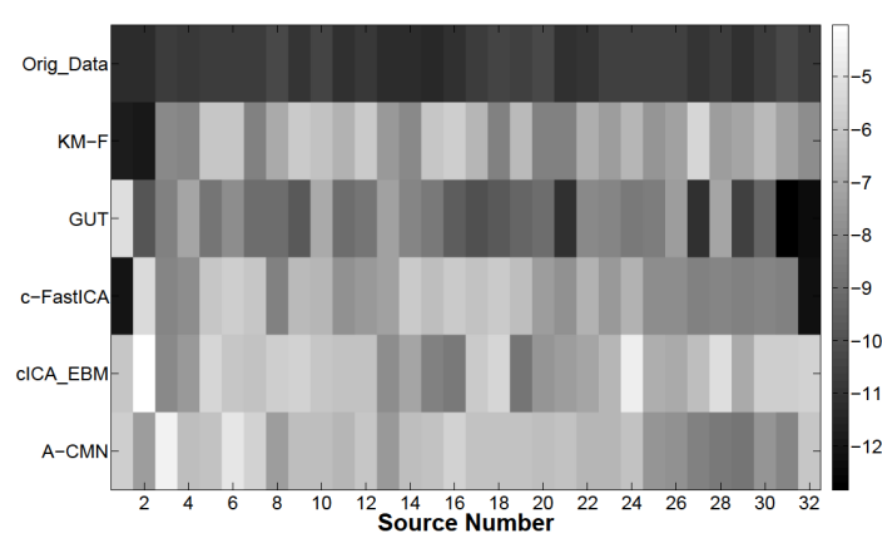

Fig. 5. Source-algorithm 2D image plot of the sparseness criterion. Each of the small rectangular areas in the first row (marked with Orig_Data) represents the sparseness for one raw EEG data. Likewise, those marked with A-CMN, cICAEBM, c-FastICA, GUT, and KM-F represents the sparseness for one estimated IC. The whiter the rectangle color is, the sparser is the IC or data channel.

whereas the other five rows represent the sparseness of the estimated 32-source from the other five algorithms. The image demonstrates how good is the separation of the original data that is achieved by the algorithms. We have mentioned earlier that the larger the sparseness index means that the source is better estimated. In this $2 \mathrm{D}$ image plot, the amount of sparseness is color-coded such that the whiter the rectangle color is, the sparser is the IC or data channel. It is clear that cICA-EBM and A-CMN achieved much better results than the other algorithms, where the degrees of sparseness for all the estimated sources are much larger than of the original data. On the other hand, KM-F and cFastICA failed in estimating IC1 and IC2, and IC1 and IC32, respectively. GUT recovered the majority of the sources with very low degrees of sparseness. Note that all the results were normalized before putting them into the image.

\section{Discussion of Results}

From the preceding shown results and the detailed ones which are impossible to be shown in this paper, we find that cICA-EBM and A-CMN outperform other algorithms, especially cICA-EBM which had performance even a bit better than A-CMN in estimating sparser components. Generally, both were able to estimate sources characterizing with: very low degree of dependence, within the range of cerebral EEG frequency bands, and clean electrical potential distribution on scalp maps.
Both c-FastICA and KM-F achieved almost the same performance as the previous ones. However, they both were also able to estimate sources with a very low degree of dependence and within the range of resting-state EEG frequency bands except some of the components which exhibit larger frequency bands which may represent some artifactual activities. Moreover, the potential distribution for most of the estimated sources was localized and dipole-like, however, still cICA-EBM and A-CMN estimated even more localized scalp maps.

The performance of GUT was the worst among the applied algorithms. The separation degree of the estimated sources was not as good as those estimated by other algorithms, where there exist some correlations between the sources that are found by GUT. Sometimes, those correlations become even higher than the correlations between the data channels themselves. GUT was able to estimate sources located within the EEG frequency bands but almost all the sources have a significant peak at high frequencies and this may have the effect of getting very similar scalp maps.

The second-order complex ICA algorithms, such as GUT that performs ICA by joint diagonalization of the covariance and pseudo-covariance matrices, impose that all the sources have different spectral coefficients which means that at most one original source can have circular distribution. This may be considered as a limitation of this algorithm. Moreover, the main work of GUT is to transform the data into uncorrelated components, whereas we always aim to get as much as possible independent components throughout applying ICA.

The KM-F algorithm utilizes kurtosis or the fourth-order cumulant for measuring the degree of Gaussianity of sources in order to optimize the separation process and it also requires that at most one source has to vanish fourth-order statistics. The c-FastICA algorithm achieved slightly worse separation than both cICA-EBM and A-CMN, this may be caused by its parallel separation of the ICs. The better performance achieved by the A-CMN algorithm may arise from its usage to the ideal nonlinearity function in the information theoretic framework for dealing with complex sources, the function uses the real and imaginary parts of the 


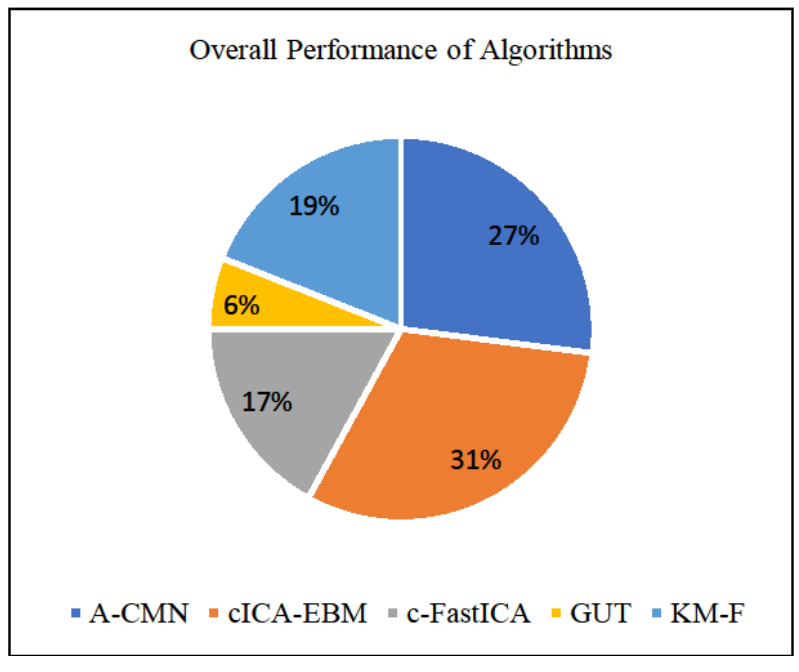

Fig. 6. Illustration of overall abilities of the used algorithms.

source for computing the log of their joint probability density.

The preceding algorithms have a common limitation in matching precise simple bivariate distributions only, therefore, they may have a limitation in the separation of sources that have a richer class of distribution. This is not the case with the cICA-EBM algorithm which showed superior separation performance due to its ability to match a wide range of bivariate distributions. Fig. 6 shows a summary of the overall performance of the used algorithms in finding sparser sources and localizing dipoles for the estimated ICs.

\section{Conclusion}

Complex-valued signals often occur in applications such as communications, radar, images, and biomedicine. Complex-valued ICA has been widely dealt with in research and practical work during the last decade. In this study, we were interested in analyzing rest-state EEG data using complex-valued ICA and then comparing the performance of different algorithms based on some criteria.

We recorded and analyzed EEG data from 105 subjects using either 22 or 32 recording electrodes in order to examine the robustness of the analysis and algorithms. The subjects were in rest-state i.e. with eyes closed and as less as possible movements in the body parts. Then Short-time Fourier transform has been used as a time/frequency analysis tool in order to exploit the propagation delay for the cerebral activity to be recorded via the electrodes, this delay was presented as phase information inherent in the EEG data. We used five complex-valued ICA algorithms based on different mathematical principles for analyzing the data.

In our study, the ground truth of the real EEG data is unknown to us, i.e. the real structure of the underlying sources in the real EEG data and the way they have been mixed are unknown. Hence, it is necessary to use some criteria that do not depend on the mixing process (mixing matrix) but rather depend on the estimated sources. So, we used three comparison criteria to assess the performance of the different algorithms. The first criterion was for assessing the degree of separating the mixed data into its original sources, fourth order measure of statistical dependency has been used to compute the correlation among the estimated sources. The second criterion was to assess the correctness of dipoles localization for the estimated sources, the residual variance between the estimated IC scalp map and the dipole scalp projection has been used for that. The third criterion was to determine whether an estimated source of being related to a brain activity or to an artifact activity, sparseness has been used to find components with the highest supergaussianity.

The obtained results showed that the performance of cICA-EBM was superior over the other algorithms. This algorithm was able to estimate sources with very low correlations and present localized scalp maps. The performance that is achieved by A-CMN was almost the same as in the case of cICA-EBM but the latter found ICs with lower sparseness values. The performance of cFastICA and KM-F was very similar, they both were also able to find ICs with very low correlation but some of the components had larger frequency bands than the normal rest-state EEG frequency bands. Such components may represent some artifactual activities. The performance of GUT was the worst; where there exist some correlations between the estimated ICs and sometimes these correlations become even higher than the correlations between the data channels themselves. 


\section{REFERENCES}

[1] A. Hyvärinen, J. Karhunen, and E. Oja, Independent Component Analysis, Illustrate. Wiley, 2001.

[2] M. Castella and J.-C. Pesquet, "An Iterative Blind Source Separation Method for Convolutive Mixtures of Images," Indep. Compon. Anal. Blind Signal Sep., vol. 3195, pp. 922-929, 2004.

[3] T. P. Jung et al., "Removing electroencephalographic artifacts by blind source separation.," Psychophysiology, vol. 37, no. 2, pp. 163-178, Mar. 2000.

[4] A. Al-Saegh, "Independent component analysis for separation of speech mixtures: a comparison among thirty algorithms," Iraqi J. Electr. Electron. Eng., vol. 11, no. 1, pp. 1-9, 2015.

[5] M. L. Scheuer, "Continuous EEG monitoring in the intensive care unit.," Epilepsia, vol. 43 Suppl 3, pp. 114-127, Jan. 2002.

[6] P. L. Nunez and R. Srinivasan, Electric Fields of the Brain: the neurophysics of EEG, 2nd ed. Oxford University Press, 2006.

[7] J. Anemüller, T. J. Sejnowski, and S. Makeig, "Complex independent component analysis of frequency-domain electroencephalographic data.," Neural Netw., vol. 16, no. 9, pp. 1311-1323, Nov. 2003.

[8] A. Hyvärinen, P. Ramkumar, L. Parkkonen, and R. Hari, "Independent component analysis of short-time Fourier transforms for spontaneous EEG/MEG analysis.," Neuroimage, vol. 49, no. 1, pp. 257-271, Jan. 2010.

[9] M. Novey and T. Adali, "Adaptable Nonlinearity for Complex Maximization of Nongaussianity and a Fixed-Point Algorithm," 2006 16th IEEE Signal Process. Soc. Work. Mach. Learn. Signal Process., no. x, pp. 79-84, Sep. 2006.

[10] X. Li and T. Adali, "Complex Independent Component Analysis by Entropy Bound Minimization," vol. 57, no. 7, pp. 1417-1430, 2010.

[11] H. Li and T. Adali, "A class of complex ICA algorithms based on the kurtosis cost function.," IEEE Trans. Neural Netw., vol.
19, no. 3, pp. 408-420, Mar. 2008.

[12] E. Ollila and V. Koivunen, "Complex ICA using generalized uncorrelating transform," Signal Processing, vol. 89, no. 4, pp. 365377, Apr. 2009.

[13] J. Eriksson and V. Koivunen, "Complexvalued ICA using second order statistics," in Machine Learning for Signal Processing, 2004. Proceedings of the 2004 14th IEEE Signal Processing Society Workshop, 2004, pp. 183-192.

[14] A. Hyvärinen and E. Oja, "Independent component analysis: algorithms and applications.," Neural Netw., vol. 13, no. 4-5, pp. 411-430, 2000.

[15] J. Onton and S. Makeig, "Information-based modeling of event-related brain dynamics.," Prog. Brain Res., vol. 159, pp. 99-120, Jan. 2006.

[16] R. Oostenveld and P. Praamstra, "The five percent electrode system for high-resolution EEG and ERP measurements.," Clin. Neurophysiol., vol. 112, no. 4, pp. 713-719, Apr. 2001. 